\title{
A Fire Alarm Sensor Node with Long Battery Life by Zero Standby Power Consumption
}

\author{
Toru Kuwatani, Yasunobu Takaichi, and Akira Yamawaki* \\ Kyushu Institute of Technology, 1-1 Sensui Tobata, Kitakyushu 804-8550, Japan \\ *Corresponding Author: yama@ecs.kyutech.ac.jp
}

\begin{abstract}
To alleviate the battery life problem for the sensor node in the wireless sensor network (WSN), we have proposed the sensor node architecture with the zero-standby power. This sensor node is suitable to detect the sudden and unexpected events like the disasters (earthquake, tsunami, landslide, flood, fire, and so on). This paper shows the fire detecting sensor node employing our proposed architecture, the silent fire detector (SFD). The SFD monitors the fire by the peltier device and never consumes any power while monitoring the fire since the ground line is disconnected by the MOSFET. This feature helps to extend the battery life of the sensor node to use the battery power fully when alerting. The MOSFET is turned on by the electromotive force from the peltier device when the fire occurs on. The SDF woken up alerts the fire to the WSN accurately and correctly by using the stable and strong power from the battery until the sensor node dies by fire. We develop the prototype hardware and demonstrate the practical execution of the SDF.
\end{abstract}

Keywords: fire alert, sensor network, battery life, peltier device.

\section{Introduction}

The many researchers have though that the wireless sensor network (WSN) is a suitable technology to monitor, detect and alert the disasters like earthquake, tsunami, landslide, flood, fire, and so on ${ }^{(1-2)}$. This is because many sensor nodes with battery have only to be put the area to be monitored without any facilitations for the power supply. However, the battery life problem of the sensor node disturbs the wildly spread usage of the WSN. To alleviate the battery life problem, several battery less sensor nodes have been proposed ${ }^{(3)}$. However, they cannot communicate on the
WSN with rich functionality like WiFi and the long distance. This is because the battery less sensor node employs an energy harvester instead of the battery. The energy harvester cannot generate the enough power to make the WSN stable according to the environment and the capacity of its own.

To make the battery life longer, the standby power reduction technologies have been proposed like the sleep mode of the microcontrollers and the wireless modules used in the sensor node ${ }^{(4)}$. Although the microcontroller and the wireless module can reduce their own power greatly, the sensor node consisting of the several components cannot eliminate the standby power completely. Empirically, the sensor nodes developed have an expected current leakage paths making the standby current from several hundred micro ampere to several mill ampere ${ }^{(5-6)}$ although the catalogue values say each component consumes several micro ampere in standby mode.

In contrast, we have proposed the sensor node architecture can eliminate the standby power completely for any organization of the sensor node ${ }^{(7)}$. This architecture can extend the battery life from 6 to 50 times, compared with the conventional sensor node (6). However, due to the characteristic feature of our sensor node, the suitable WSN applications for our node have been enough shown. We have only demonstrated the door monitoring system ${ }^{(5)}$.

This paper shows the sensor node employing our proposed architecture monitoring, detecting and alerting the fire which is one of the significant disasters. We call this

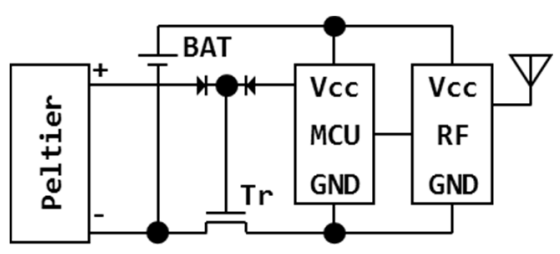

Fig. 1. Sensor Node Architecture 


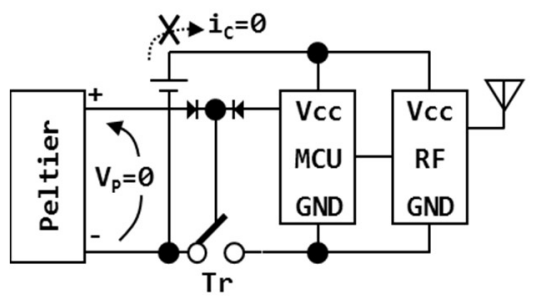

(a) Fire monitoring mode Sensor node is standby.

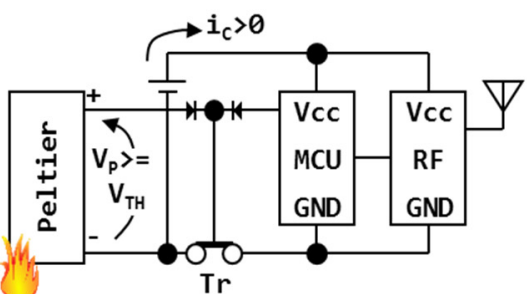

(b) Fire detecting mode Sensor node wakes up.



(c) Fire alerting mode

Sensor node sends alert to WSN.

Fig. 2. Sensor Node Execution

sensor node the silent fire detector (SFD). We develop the prototype hardware and demonstrate the practical execution of the SDF. Since the SDF never uses the battery power in monitoring fire, the SDF can alert the fire communicating with long distance by using strong and stable power on the almost full discharged battery.

The rest of the paper is organized as follows. Section 2 describes the architecture of the SDF and its execution. Section 3 develops the prototype hardware realizing the SDF. Section 4 shows the experimental results and discusses them. Finally, Section 5 concludes the paper with future work.

\section{Silent Fire Detector}

\subsection{Sensor Node Architecture}

Fig. 1 shows the fire detecting sensor node employing our proposed architecture, the silent fire detector (SFD). The SFD monitors the fire by the peltier device and never consumes any power when monitoring the fire since the ground line is disconnected by the MOSFET. This feature helps to extend the battery life of the sensor node to use the battery power fully when alerting. The MOSFET is turned on by the electromotive force from the peltier device when the fire occurs on. The SDF woken up alerts the fire to the WSN accurately and correctly by using the stable power from the battery until the sensor node dies by fire.
The SDF does not need to cut the leakage paths across any kinds of components over the sensor node. The products employing the SDF have only to cut the ground line by a single transistor such like closing the main valve of water.

\subsection{Sensor Node Execution}

Fig.2 shows the execution snapshot of the SFD. When monitoring the fire, the SFD does not consume any power from the battery as shown in Fig.2 (a); Fire monitoring mode. Since the power transistor disconnects the ground line, there is no closed loop the consumed current flows. This is most characteristic feature which can extend the battery life significantly while monitoring the fire.

When the fire occurs on the peltier device, the peltier device generates the electromotive force and turns on the power transistor as shown in Fig. 2 (b); Fire detecting mode. The consumed current begins to flow and the sensor node wakes up. Until the fire detecting mode, the battery has never been consumed.

The sensor node completely waken up drives the power transistor by some port with the stable high voltage nearly $\mathrm{V}_{\mathrm{CC}}$ to keep execution correctly instead of the swinging output of the peltier device. The sensor node establishes the WSN and alerts the fire to the WSN by fully using the battery power. The enough power from the battery makes the WSN stable and succeeds the correct communication as shown in Fig.2 (c); Fire alerting mode.

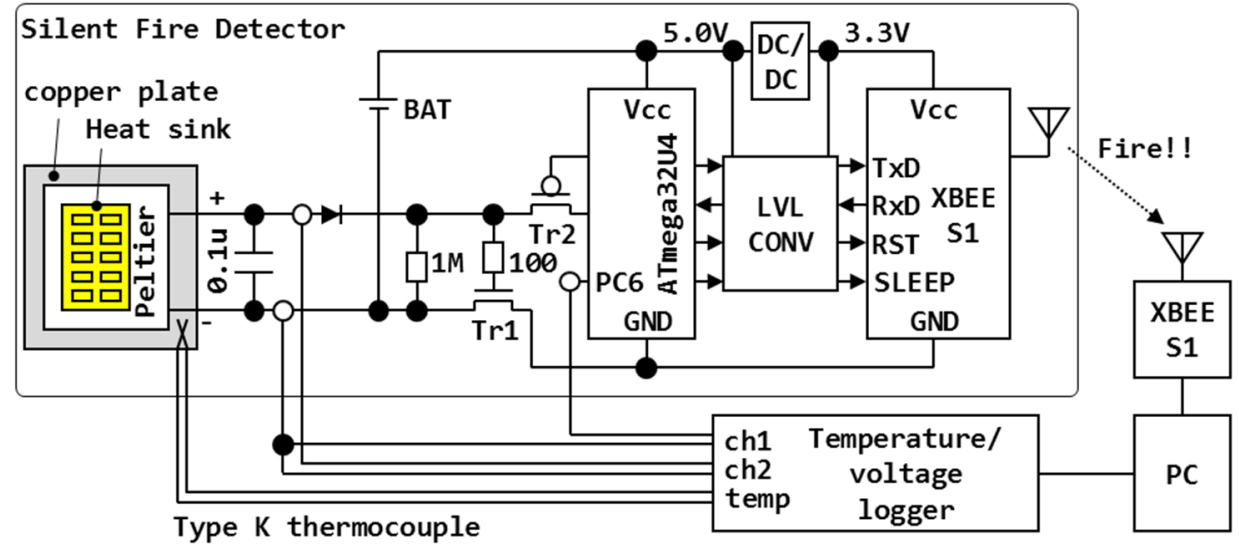

Fig. 3. Prototype Hardware 


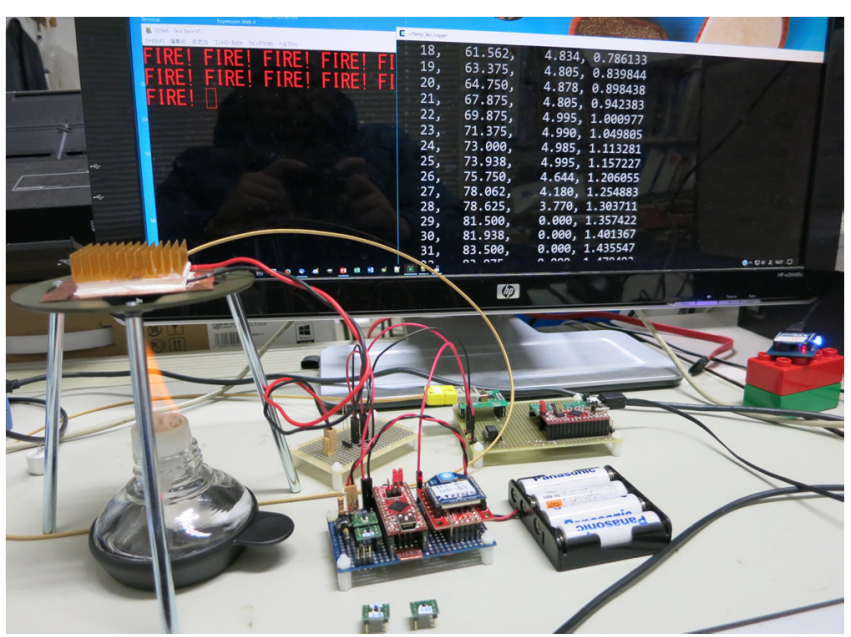

Fig. 4. Picture of Experimental Setup

\section{Prototype Hardware}

Fig. 3 depicts the block diagram of the prototype hardware.

In the SDF, the peltier device is the TEC1-12706. To strengthen the electromotive force caused by the difference in temperature between the cold side and hot side of the peltier device, we attached the heat sink to the cold side and the cupper plate to the hot side. As the microcontroller, we used the Atmega32u4. The wireless module was the XBEE $\mathrm{S} 1$. Since the XBEE needs $5 \mathrm{~V}$ power supply and the Atmega32u4 needs $3.3 \mathrm{~V}$, we inserted the level converter between $5 \mathrm{~V}$ and $3.3 \mathrm{~V}$ based on the N-type transistor into the boundary of the XBEE and the Atmega32u4.

$\operatorname{The} \operatorname{Tr} 1$ is the power transistor to disconnect the ground line when the sensor node is the fire monitoring mode. In the fire monitoring mode, all pins of the microcontroller are pulled up to Vcc because the ground is floated. Thus, the Tr2 cuts the connection between the gate of the $\operatorname{Tr} 1$ and the port pin of the microcontroller. By this, the gate of the Tr1 is pulled down to the ground through the $1 \mathrm{M} \Omega$ register in the fire monitoring mode.

To measure the temperature of the peltier device, and observe the output voltages of the peltier device and test pin from the microcontroller (PC6), we developed a temperature / voltage logger. The logger uses the MPC9600 to measure the temperature by the type $\mathrm{K}$ thermocouple. The A/D converters on the Atmega32u4 are used to measure the voltages of the electromotive force from the peltier device and test pin from the microcontroller. The logged data is stored into the personal computer via the USB. The sampling period is about $500 \mathrm{~ms}$.

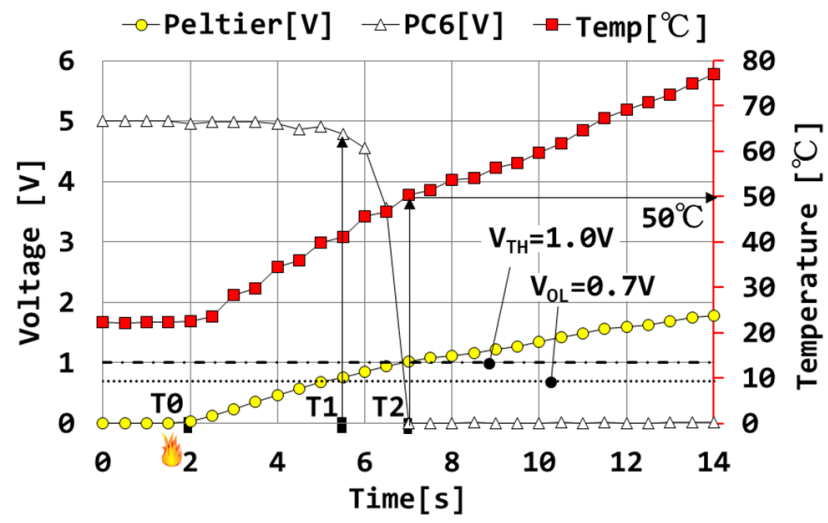

Fig. 5. Experimental Result $\mathrm{V}_{\text {тн }}$ : Min. 0.45V, Typ. 0.65V, Max. 1.0V

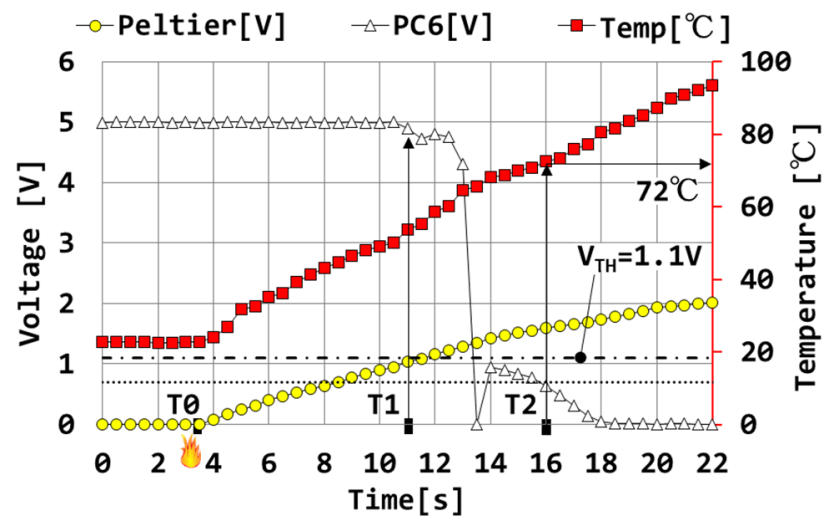

Fig. 6. Experimental Result

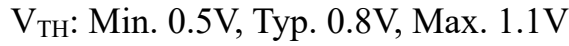

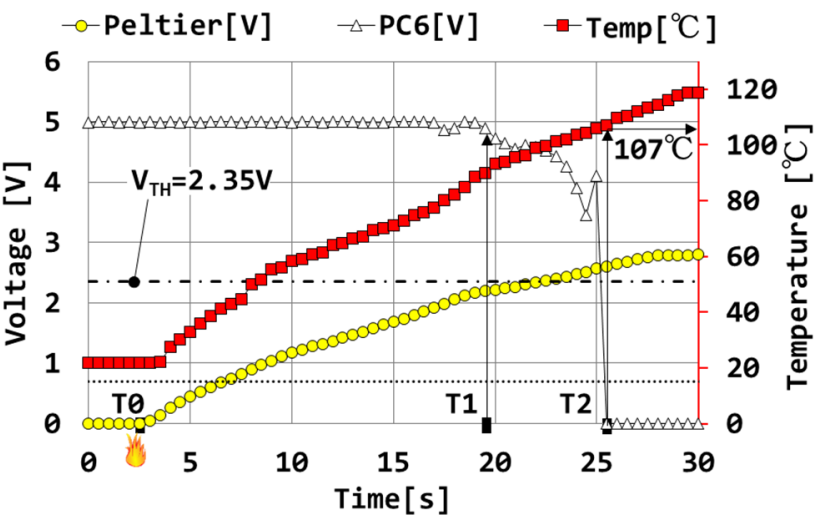

Fig. 7. Experimental Result

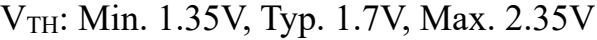

\section{Experiment}

Fig. 4 shows the experimental setup. To burn the peltier device, we used an alcohol lamp as shown in Fig. 4.

Fig. 5, Fig. 6 and Fig. 7 show the experimental results according to the used power transistors with different gate threshold voltages. The Tab. 1 shows the used transistors and 
Tab. 1 Used Transistors

\begin{tabular}{l|ccc}
\hline \multirow{2}{*}{ Transistor } & \multicolumn{3}{|c}{ VTH[V] } \\
\cline { 2 - 4 } & Min. & Typ. & Max. \\
\hline PJE260N02 & 0.45 & 0.65 & 1.00 \\
IRLML6344PbF & 0.50 & 0.80 & 1.10 \\
IRFML8244TRPbF & 1.35 & 1.70 & 2.35 \\
\hline
\end{tabular}

their threshold voltages.

The T0 in Fig. 5 to Fig. 7 indicates the time when we put the fire on the alcohol lamp. The microcontroller sets the test pin (PC6) to zero after waking up. The test pin pulled up to $\mathrm{Vcc}$ in the fire monitoring mode is going down to the ground level when the sensor node enters the fire detecting mode. The T1 means the time when the electromotive force of the peltier device is getting closer to the maximum gate threshold voltage $\left(\mathrm{V}_{\mathrm{TH}}\right)$ and the test pin of the microcontroller is going down to the ground level. That is, the sensor node moves to the fire detecting mode. The T2 indicates the time when the test pin becomes lower than the $\mathrm{V}_{\mathrm{OL}}(0.7 \mathrm{~V})$ defined by the specification of the microcontroller. That is, the microcontroller performs the execution stably and is ready to send the alert to the WSN. We think the sensor node enters the fire alerting mode at T2. Also, we have confirmed that the personal computer correctly got the alert from the sensor node.

Those experimental results indicate that our proposal can be implemented as the real fire detecting device because the test pin goes down to the ground level.

As shown in the experimental results, the times of entering to the fire alerting mode are differ according to the gate threshold voltages used transistors. The transistor with low gate threshold voltage alerts the fire when the temperature of about $50^{\circ} \mathrm{C}$. The middle threshold alerts at $72^{\circ} \mathrm{C}$ and the high threshold alerts at $107^{\circ} \mathrm{C}$. Thus, we can control the temperature to alert the fire as similar to the sprinklers widely used.

\section{Conclusions}

To alleviate the battery life problem for the sensor node in the wireless sensor network (WSN), we have proposed the sensor node architecture with the zero-standby power.

This paper shows the fire detecting sensor node employing our proposed architecture, the silent fire detector (SFD). The SFD monitors the fire by the peltier device and never consumes any power when monitoring the fire since the ground line is disconnected by the MOSFET. This feature helps to extend the battery life of the sensor node.

We have developed the prototype hardware and demonstrate the practical execution of the SDF. As a result, we have confirmed that our proposal can be implemented as the real fire detecting device. Also, by using the transistors with different gate threshold voltages, our proposal can control the temperature to alert the fire as similar to the sprinklers widely used.

As future work, we will investigate the more applications of the WSN employing our proposal.

\section{Acknowledgment}

This work was supported by JSPS KAKENHI Grant Number 26330106.

\section{References}

(1) J.f. Tu, Y. Yang, C. Li, A. He, and L. Li: "A Contextadaptive and Energy-efficient Wireless Sensor Network for Debris Flow Monitoring", International Conference on Wireless Communication and Sensor Network, pp. 157-161, 2014.

(2) G. Hirakawa, P. P. Kywe, K. Ito, and Y. Shibata: "Automotive Sensor Network Platform for Disaster Information System", 28th International Conference on Advanced Information Networking and Applications Workshops, pp.623-626, 2014.

(3) G. Zhou, L. Huang, W. Li, and Z. Zhu, "Harvesting Ambient Environmental Energy for Wireless Sensor Networks: A Survey," Journal of Sensors, Vol. 2014, Article ID 815467, 20 pages (2014)

(4) K. P. Naveen and A. Kumar, "Relay Selection for Geographical Forwarding in Sleep-Wake Cycling Wireless Sensor Networks," IEEE Transactions on Mobile Computing, Vol. 12, No. 3, pp. 475-488 (2013)

(5) A. Yamawaki, S. Serikawa, "Applying sensor node with zero standby power to door monitor", Lecture Notes in Engineering and Computer Science: Proceedings of The International MultiConference of Engineers and Computer Scientists 2016, pp. 576-580 (2016)

(6) A. Yamawaki, S. Serikawa, "Battery Life Estimation of Sensor Node with Zero Standby Power Consumption", International Conference on Computational Science and Engineering, pp.166-172 (2016)

(7) A. Yamawaki, M. Yamanaka and S. Serikawa: "A sensor node architecture with zero standby power on wireless sensor network", Artificial Life and Robotics, vol. 20, no. 3, pp.210-216 (2015) 